Check for updates

Cite this: Phys. Chem. Chem. Phys., 2018, 20, 5399

Received 31st August 2017, Accepted 10th November 2017 DOI: $10.1039 / c 7 c p 05957 d$

rsc.li/pccp

\title{
Dissociative ionisation of adamantane: a combined theoretical and experimental study $\dagger$
}

\author{
Alessandra Candian, (D) *a Jordy Bouwman, (iD $\star^{*}{ }^{\mathrm{b}}$ Patrick Hemberger, (iD c \\ Andras Bodi iD ${ }^{c}$ and Alexander G. G. M. Tielens ${ }^{a}$
}

\begin{abstract}
Diamond nanoparticles, or nanodiamonds, are intriguing carbon-based materials which, maybe surprisingly, are the most abundant constituent of presolar grains. While the spectroscopic properties of even quite large diamondoids have already been explored, little is known about their unimolecular fragmentation processes. In this paper we characterise the dissociative ionisation of adamantane $\left(\mathrm{C}_{10} \mathrm{H}_{16}\right)$ - the smallest member of the diamondoid family - utilising imaging Photoelectron Photoion Coincidence (iPEPICO) spectroscopy and Density Functional Theory (DFT) calculations. We have found adamantane to dissociatively photoionise via several parallel channels of which $\mathrm{H}, \mathrm{C}_{3} \mathrm{H}_{7}$ and $\mathrm{C}_{4} \mathrm{H}_{8}$ losses are the most important ones. Calculations confirm the existence of a rate-limiting transition state for the multiple C-loss channels, which is located at $10.55 \mathrm{eV}$ with respect to neutral adamantane. In addition, we found dissociation channels leading to small cationic hydrocarbons, which may be relevant in the interstellar medium.
\end{abstract}

\section{Introduction}

Diamondoids are a class of nanometer-sized hydrocarbons that have a diamond-like fused ring structure, with adamantane $\left(\mathrm{C}_{10} \mathrm{H}_{16}\right)$ being the smallest unit cage. The diamond-like structure results in a remarkable rigidity, strength, and thermodynamic stability; properties that make them intriguing subjects of study for chemists and physicists alike. ${ }^{1,2}$ On Earth, small diamondoids are found in natural gas reservoirs and are abundant in gas pipeline deposits. ${ }^{3}$ Recently, larger diamondoids have been isolated from petroleum, ${ }^{4}$ triggering experimental studies to elucidate the properties of these molecules that bridge the gap between hydrocarbons and nano-sized diamond crystals.

The discovery of small $(2-3 \mathrm{~nm})$ nanodiamonds in meteorites ${ }^{5}$ drew the attention of astronomers and geologists to this material. It is now established that nanodiamonds are the most abundant component of presolar grains. ${ }^{6,7}$ Due to their high stability, diamond-like material is also expected to be present and

\footnotetext{
${ }^{a}$ Leiden Observatory, Leiden University, P.O. Box 9513, 2300-RA Leiden,

The Netherlands. E-mail: candian@strw.leidenuniv.nl

${ }^{b}$ Radboud University Nijmegen, Institute for Molecules and Materials,

FELIX Laboratory, Toernooiveld 7c, NL-6525 ED Nijmegen, The Netherlands. E-mail: bouwman@strw.leidenuniv.nl

${ }^{c}$ Laboratory for Synchrotron Radiation and Femtochemistry, Paul Scherrer Institute, CH-5232 Villigen-PSI, Switzerland

$\dagger$ Electronic supplementary information (ESI) available: Full PES of observed dissociation channels, structures of intermediate and transition states, cartesian coordinates of the rate limiting transition states. See DOI: $10.1039 / \mathrm{c} 7 \mathrm{cp} 05957 \mathrm{~d}$ \# Present address: Sackler Laboratory for Astrophysics, Leiden Observatory, Leiden University, P.O. Box 9513, 2300-RA Leiden, The Netherlands.
}

abundant in the interstellar medium. ${ }^{8,9}$ Guillois et al. ${ }^{10}$ attributed two broad emission bands at 3.45 and $3.53 \mu \mathrm{m}$, observed in the spectrum of circumstellar disks around the stars Elias 1 and HD 97048 to large $(50 \mathrm{~nm})$ gas-phase diamond-like molecules. Later, small nano-diamondoid molecules with sizes up to $130 \mathrm{C}$ were shown to reproduce the above features very well. ${ }^{11,12}$ The size of astronomical nanodiamonds is still a matter of debate and so is the reason why only a handful of astronomical sources have been found to exhibit diamondoid spectral features. Nevertheless, based on the strength of the emission bands, it was proposed that the amount of cosmic carbon locked in nanodiamonds is of the order of $1-2 \% .^{13}$

A considerable number of investigations have been devoted to diamondoids in the laboratory, too. The IR spectra of large neutral diamondoids (up to hexamantane, $30 \mathrm{C}$ ) have been recorded experimentally. ${ }^{14}$ Vibrational and electronic spectra for a sample of both neutral and cationic species were calculated ${ }^{13}$ and ionisation potentials were determined experimentally. ${ }^{15}$ More recently, a comprehensive study of the electronic spectroscopy of adamantane, diamantane and triamantane was performed by Steglich et al. ${ }^{16}$ As expected for aliphatic compounds, the photoionisation of the diamondoids may result in the loss of atomic hydrogen, especially at low energies. The gas-phase infrared spectrum of the adamantyl cation $\left(\mathrm{C}_{10} \mathrm{H}_{15}{ }^{+}\right)-$the fragment resulting from the $\mathrm{H}$-loss of adamantane - was recorded and, based on a comparison with quantum chemical calculations, it was found to be consistent with the lowest energy isomer, 1-adamantyl. ${ }^{17} \mathrm{Lu}$ et al. $^{18}$ computed the ionisation potential of diamondoids and proposed dissociation paths for 
neutrals and cations, and suggested that the products predominantly consist of small hydrocarbons. The dissociation of adamantane and diamantane was also studied experimentally in an electron impact mass spectrometer. ${ }^{19}$ It was suggested that, analogous to pyrolysis reactions, i.e. dissociation on the neutral surface, the dissociative ionisation of diamondoids yields mostly benzene derivatives.

While experiments have been conduced to explain the very specific local conditions that may lead to the paucity of nanodiamonds in space, ${ }^{20}$ little is known about the photodissociation behaviour of nanodiamonds. If these species were easily destroyed by photons, this would be an additional explanation for the lack of detection of their emission spectra. Thus, here we aim to characterise the dissociative photoionisation of the smallest diamondoid, adamantane $\left(\mathrm{C}_{10} \mathrm{H}_{16}\right.$, Fig. 1), by studying the branching ratios and fragmentation rate constants of internal energy selected parent ions experimentally and exploring the potential energy surface (PES) computationally. The results from quantum chemical calculations are used as input for a supporting statistical dissociation model. The products formed from the photodissociation as well as the underlying formation mechanisms are rationalised and $0 \mathrm{~K}$ appearance energies are extracted. The findings are discussed in light of their astrophysical relevance.

\section{Methods}

\subsection{Experimental method}

Imaging photoelectron photoion coincidence (iPEPICO) experiments have been performed at the Vacuum Ultraviolet (VUV) beamline of the Swiss Light Source. The beamline and the iPEPICO instrument have been discussed in detail elsewhere ${ }^{21,22}$ and will only be described briefly here.

Adamantane (99\%, Fig. 1) was purchased from Sigma-Aldrich and was used without further purification. The adamantane sample was kept at room temperature and its vapour was introduced into the detection chamber through a Teflon tube. The effusive adamantane beam intersects with the VUV light from the beamline in the detection chamber, which is kept at a pressure of $5 \times 10^{-7}$ mbar. The VUV radiation is generated in a bending magnet, is dispersed on a 600 groove $\mathrm{mm}^{-1}$ grating

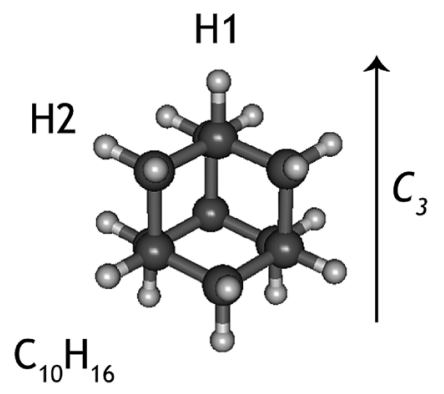

Fig. 1 Structure of the adamantane molecule $\mathrm{C}_{10} \mathrm{H}_{16}$. The arrow shows the $C_{3}$ rotation axis along which the adamantyl cation deforms, due to Jahn-Teller effect. $\mathrm{H} 1$ and $\mathrm{H} 2$ identify two non-equivalent $\mathrm{H}$ atoms in the cations. and is focused on the exit slit where the experimental chamber is situated. The light is passed through a noble gas filter before entering the experimental chamber to remove high order radiation.

Photoelectrons and photoions that result from the VUV induced (dissociative) ionisation are extracted in a $120 \mathrm{~V}$ $\mathrm{cm}^{-1}$ electric field. Electrons are velocity map imaged onto a RoentDek delay line imaging detector and their arrival time serves as time zero for measuring the time of flight of the coincident ions. The photoions are space focused in a WileyMcLaren time-of-flight (TOF) tube onto a chevron microchannel plate detector. The flight time yields the mass of the coincident ion. The long first acceleration region at a relatively low field results in ion residence times on the order of several microseconds. If the dissociation is not prompt, the fragment ions will only have a time-dependent fraction of the nominal kinetic energy, which leads to a longer time of flight and shows up as an asymmetric daughter ion peak, broadened towards higher TOF. The peak shape yields information on the total depletion rate of the parent ion. The mass spectrometer is designed such that a compromise is found between the mass resolution $(m / \Delta m \approx 150)$ and the low fields needed to resolve the dissociation of metastable ions. Dissociation rates in the $10^{3}<k(E) / \mathrm{s}^{-1}<10^{7}$ can be measured in this configuration, and can then be extracted taking into account the ion optics parameters. $^{23}$

Threshold photoelectrons, i.e. electrons with close to zero kinetic energy, in our case $E \leq 0.01 \mathrm{eV}$, are imaged onto the center of the detector with a kinetic energy resolution better than $1 \mathrm{meV}$. Contributions by electrons with non-zero kinetic energy but no lateral momentum, which are also imaged onto the center, have been subtracted as proposed in the literature. ${ }^{24}$ The threshold photoelectron spectrum is thus obtained by recording this signal as a function of photon energy. Selecting only threshold electrons for coincidences enables internal energy selection of the parent ion. By scanning the monochromatic VUV radiation the dissociation process can now be accurately explored.

The fractional parent and daughter ion abundances in threshold photoionisation are plotted in the breakdown diagram (BD). In parallel processes, the BD is indicative of the branching ratio of the different channels, whereas the asymmetrically broadened daughter ion peaks in mass spectrum reflect the total dissociation rate of the parent ion.

\subsection{Computational methods}

Theoretical calculations were performed using Density Functional Theory (DFT) methods with B3LYP/6-311++G(2d,p) and $\omega$ B97X-D/ $6-311++G(2 d, p)$ using Gaussian $09 .{ }^{25}$ The reactant, product and intermediate structures were optimised and vibrational analysis was performed to confirm that they are local minima on the potential energy surface (absence of imaginary frequencies). Transition states were evaluated using the Synchronous TransitGuided Quasi-Newton (STQN) method, ${ }^{26,27}$ which requires the geometry of reactants, products and, optionally, an initial guess for the transition state geometry. The transition state nature of the 
structure was then confirmed through vibrational analysis by the presence of one imaginary frequency. To analyse the results of the calculations, the visualisation program GABEDIT ${ }^{28}$ was used.

The optimised geometry and normal modes of the neutral and ionic ground state of adamantane computed at the $\omega \mathrm{B} 97 \mathrm{X}-\mathrm{D} /$ 6-311++G(2d,p) level of theory are used as input for a FranckCondon simulation of the adamantane photoelectron spectrum. The eZspectrum software package ${ }^{29}$ was used to generate a stick diagram of the spectrum, which was subsequently convoluted with a 20 meV Full-Width-at-Half-Maximum (FWHM) Gaussian peak profile to account for the experimental resolution and the rotational envelope at room temperature.

A statistical model was constructed to account for the fractional ion abundances in the $\mathrm{BD}$ and the unimolecular dissociation rates measured in the form of TOF peak shapes, as descibed by Sztaray et al. ${ }^{23}$ The internal energy distribution of the neutral and the density of states of the dissociating species were computed based on B3LYP/6-311G++(2d,p) vibrational frequencies and rotational constants. The same level of theory was used to calculate the number of states of the rate limiting transition states and the barrier heights thereto, also required in the Rice-Ramsperg-Kassel-Marcus (RRKM) rate equation. ${ }^{30}$ These are the fit parameters that were allowed to relax so that the model reproduces the experimental results faithfully: the three lowest frequencies of the transition state were scaled by a variable factor and the barrier heights were varied to minimise the fit error. Accurate $0 \mathrm{~K}$ appearance energies are thus retrieved based on the best fit to the breakdown diagram and time-of-flight distributions.

\section{Results}

The experimental results are described first. Next, the quantum chemical computations are discussed to rationalise the formation of the detected products. These computational results are subsequently used to model the experimental data and to derive appearance energies.

\subsection{Experimental results}

3.1.1 Threshold photoelectron spectrum. The threshold photoelectron spectrum of adamantane is recorded by scanning the photon energy from 9 to $10 \mathrm{eV}$ in steps of $13 \mathrm{meV}$, as presented in Fig. 2. A sharp resonance corresponding to the band origin is found at $(9.29 \pm 0.02) \mathrm{eV}$. This value compares well with the evaluated ionisation energy of $(9.25 \pm 0.04) \mathrm{eV}$ from the NIST database. ${ }^{31}$ A Franck-Condon simulation of the ionisation process from the neutral ground state into the ground state of the adamantane ion is also shown in Fig. 2. The simulated spectrum matches the position of the experimentally observed transitions very well. We can also identify four bands at 9.39, 9.45, 9.55, and $9.60 \mathrm{eV}$ in the experimental spectrum. According to the simulation, a ring breathing motion of the $\mathrm{C}_{6}$-ring explains the feature at $9.39 \mathrm{eV}$ (calculated frequency of $900 \mathrm{~cm}^{-1}$ ), whereas the $9.45 \mathrm{eV}$ transition is associated with a totally symmetric umbrella-like motion of the $\mathrm{C}_{4}$-unit bridging the $\mathrm{C}_{6}$-chair, with

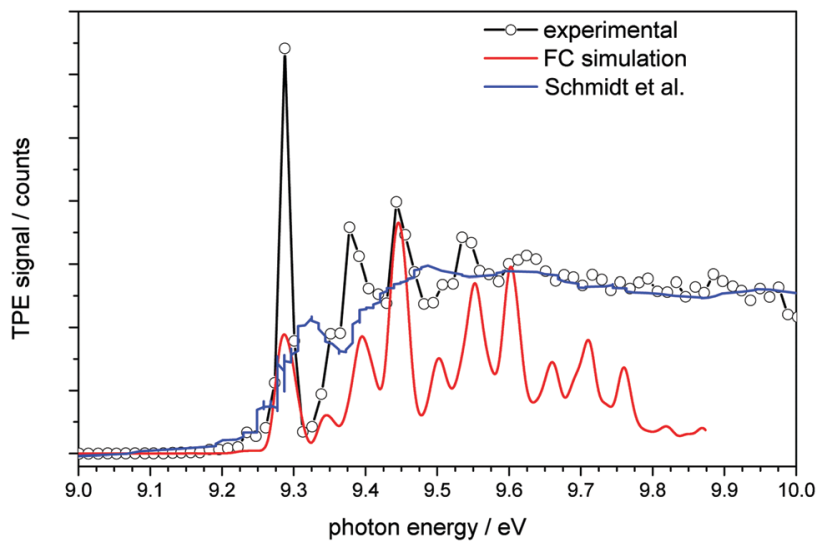

Fig. 2 Threshold photoelectron spectrum of adamantane plotted together with a simulated spectrum (red line) and the conventional photoelectron spectrum of Schmidt et al. ${ }^{32}$ (blue line).

a calculated frequency of $1284 \mathrm{~cm}^{-1}$. The bands at 9.55 and $9.6 \mathrm{eV}$ can be assigned to overtones and combination bands of these transitions. Two aspects catch the eye when comparing the simulated and experimental spectrum. First, the intensity of the $0_{0}^{0}$ transition, located at $9.24 \mathrm{eV}$ is significantly underestimated in the simulation. The relative intensity of the origin transition in the HeI photoelectron spectrum of Schmidt et al. ${ }^{32}$ (see Fig. 2, blue line) is, however in good agreement with the FranckCondon simulation. This indicates that the intensity gain in the origin transition is due to secondary ionisation processes, such as autoionisation, and not related to the Jahn-Teller distortion of the cation, i.e., the fact that neutral adamantane of $T_{\mathrm{d}}$ symmetry forms a $C_{3 \mathrm{v}}$ ground state cation upon ionisation. ${ }^{33,34}$ Second, the threshold photoelectron signal between 9.6 and $10 \mathrm{eV}$ is almost constant, in contrast with the simulation, which predicts the ground state band to drop in intensity quickly above $9.6 \mathrm{eV}$. In this energy range, the threshold and HeI photoelectron spectra agree, ${ }^{32,35}$ which implies that the close to constant signal intensity is due to favourable nuclear wave function overlap with the final ion state. Indeed, close lying electronically excited states of the ion are found at 0.07 and $1.34 \mathrm{eV}$ above the vertical ionisation energy using time-dependent DFT calculations $(\omega \mathrm{B} 97 \mathrm{X}-\mathrm{D} / 6-311++\mathrm{G}(2 \mathrm{~d}, \mathrm{p}))$.

3.1.2 Dissociative ionisation. Fig. 3 shows illustrative threshold ionisation mass spectra of adamantane at photon energies ranging from 11.10 to $11.90 \mathrm{eV}$. The thermal mass spectral peak width at the moderate field of $120 \mathrm{~V} \mathrm{~cm}^{-1}$, required because of the fragmentation rate and electron kinetic energy analysis, inherently limits the mass resolution to $m / \Delta m \approx 150$. As mentioned earlier, the long acceleration region is used to measure dissociation rates in slow fragmentations, which are essential to account for kinetic shifts. However, the resulting peak broadening further complicates signal apportioning to $m / z$ channels in the metastable energy range. Thus, the products at $m / z=95,94$, and 93 (loss of a $\mathrm{C}_{3} \mathrm{H}_{x}$ unit with $x=5,6$, and 7 ) and $m / z=81,80,79$, and 78 (loss of a $\mathrm{C}_{4} \mathrm{H}_{y}$ unit, with $y=7,8,9$, and 10) show up as a broad clusters of peaks. Within these clusters, the peaks located at $m / z=80$ and 93 corresponding to the loss of a $\mathrm{C}_{4} \mathrm{H}_{8}$ and $\mathrm{C}_{3} \mathrm{H}_{7}$ unit, respectively, are the dominating 


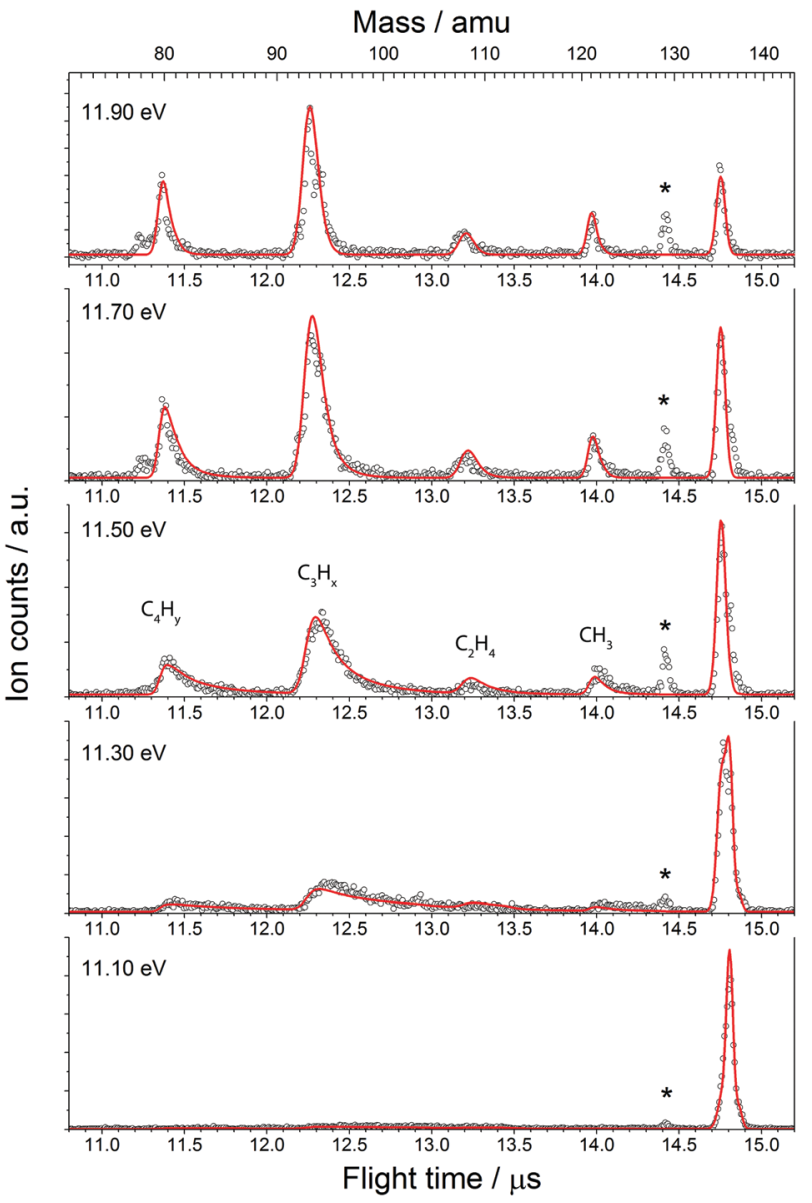

Fig. 3 Threshold ionisation mass spectra of adamantane at photon energies of 11.10, 11.30, 11.50, 11.70 and $11.90 \mathrm{eV}$ (open circles) plotted together with model fits to the data (red lines). The star $\left(^{*}\right)$ marks a contamination that is present in the system, but does not interfere with the measurements. The peaks are labeled according to the correspondent loss channel. Due to insufficient mass resolution, $\mathrm{H}$-loss peak is unresolved, see Section 3.1.3 for details.

decomposition channels. The species detected at $\mathrm{m} / \mathrm{z}=129$ corresponds to quinoline that was used in prior experiments and is known not to interfere with the experiments reported here. $^{36}$

3.1.3 Breakdown diagram. The VUV photon energy is scanned from to 10.9 to $12.1 \mathrm{eV}$ in $50 \mathrm{meV}$ steps to record TPEPICO mass spectra from which the breakdown diagram is constructed (see Fig. 3 for illustrative TOF mass spectra). The asymmetric TOF peak shapes point to a slow dissociation during the acceleration of the parent ion in the mass spectrometer, which we use to obtain rate information. The broad peak at $12.3 \mu$ s corresponds to the loss of $\mathrm{C}_{3} \mathrm{H}_{x}$-species and is the dominant channel, followed by hydrogenloss and the loss of the $\mathrm{C}_{4} \mathrm{H}_{y}$ species located at $11.4 \mu$ s. Methyl and ethene loss (peaks at 14.0 and $13.2 \mu \mathrm{s}$ ) represent minor dissociation channels with fractional abundances smaller than $10 \%$. The peaks assigned to the loss of $\mathrm{C}_{3} \mathrm{H}_{x}$ and $\mathrm{C}_{4} \mathrm{H}_{y}$ are analysed as a single dissociation channel in the $\mathrm{BD}$ analysis, because they are not resolved experimentally. The H-loss daughter ion peak and the parent ion peak are also unresolved. However, since the metastable peak broadening of the H-loss peak is negligible, the centre-ofgravity analysis of this band quantifies the individual contributions of the parent ion and the H-loss product. ${ }^{36,37}$ The center of gravity of the parent and H-loss peaks is evaluated as:

$$
\mu=\frac{\int t \cdot \operatorname{TOF}(t) \mathrm{d} t}{\int \operatorname{TOF}(t) \mathrm{d} t}
$$

where TOF stands for the time-of-flight distribution as a function of the time of flight, $t$. The contributions of the parent ion and H-loss channel are subsequently calculated according to:

$$
\mu=a t_{1}+(1-a) t_{2},
$$

with $t_{1}$ and $t_{2}$ the arrival times of the parent ion and $\mathrm{H}$-loss product ions, respectively. The factor $a$ assumes a value between 0 and 1 and reflects the relative abundance of parent ion in the combined $[\mathrm{P}]^{+}$and $[\mathrm{P}-\mathrm{H}]^{+}$TOF peak.

The $\mathrm{BD}$, constructed by plotting the integrated ion signal contributions of each dissociation product normalised to the total ion signal as a function of internal energy of the parent ion, is shown in Fig. 4. It can be seen that many of the product channels open up at approximately the same photon energy, $h \nu \approx 10.9 \mathrm{eV}$. This hints to a common rate limiting transition state for at least some of the channels, as was observed in the past for other systems as well. ${ }^{38,39} \mathrm{~A}$ summary of the parallel dissociation channels and the accompanying co-fragments is shown in Table 1.

In the breakdown diagram, there is a lingering parent ion signal at $<4 \%$ abundance in the $11.4-11.6 \mathrm{eV}$ photon energy range. This cannot be reconciled with the internal energy distribution of the neutral sample, and is most likely an artifact of the center-of-gravity analysis. While we cannot rule out competing alternative internal energy loss processes in the parent ion, such as fluorescence, it appears to be extremely unlikely that they should result in such an artifact. In the modelling, we do not consider this remaining fraction of the parent ion in the $\mathrm{BD}$ and the parent ion channel is set to zero above $11.65 \mathrm{eV}$.

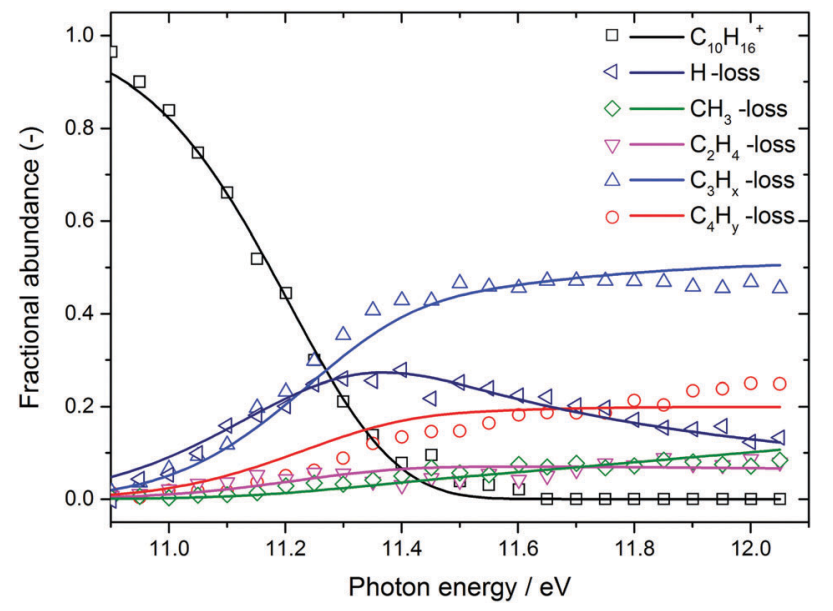

Fig. 4 Adamantane breakdown diagram (open symbols) plotted together with the RRKM model fit (solid lines). Appearance energies derived from the fits are summarised in Table 1. 
Table 1 Fragment ion peaks, the corresponding product ions and neutrals formed by (dissociative) photoionisation of adamantane with $0 \mathrm{~K}$ appearance energies (A.E.) and entropy of activation at $1000 \mathrm{~K}\left(\Delta S^{\ddagger}\right)$ derived from the RRKM model. The star * marks the strongest peak in a cluster of peaks

\begin{tabular}{|c|c|c|c|c|}
\hline$m / z$ & Product & Co-fragment & A.E. $(e V)$ & $\Delta S^{\ddagger}\left(\mathrm{K}(\mathrm{J} \mathrm{mol})^{-1}\right)$ \\
\hline 136 & $\mathrm{C}_{10} \mathrm{H}_{16}^{\bullet+}$ & - & - & - \\
\hline 135 & $\mathrm{C}_{10} \mathrm{H}_{15}{ }^{+}$ & $\bullet$ H-Loss & 10.50 & -32.30 \\
\hline 121 & $\mathrm{C}_{9} \mathrm{H}_{13}^{+}$ & ${ }^{\circ} \mathrm{CH}_{3}$-Loss & 10.83 & 11.26 \\
\hline 108 & $\mathrm{C}_{8} \mathrm{H}_{12}^{\bullet+}$ & $\mathrm{C}_{2} \mathrm{H}_{4}$-Loss & 10.69 & -22.14 \\
\hline 95 & $\mathrm{C}_{7} \mathrm{H}_{11}{ }^{+}$ & ${ }^{\bullet} \mathrm{C}_{3} \mathrm{H}_{5}$-Loss & & \\
\hline 94 & $\mathrm{C}_{7} \mathrm{H}_{10}{ }^{\bullet+}$ & $\mathrm{C}_{3} \mathrm{H}_{6}$-Loss & & \\
\hline 93 & $\mathrm{C}_{7} \mathrm{H}_{9}{ }^{++}$ & ${ }^{\bullet} \mathrm{C}_{3} \mathrm{H}_{7}-$ Loss $^{*}$ & 10.69 & 1.82 \\
\hline 81 & $\mathrm{C}_{6} \mathrm{H}_{9}{ }^{++}$ & ${ }^{\bullet} \mathrm{C}_{4} \mathrm{H}_{7}-\mathrm{Loss}$ & & \\
\hline 80 & $\mathrm{C}_{6} \mathrm{H}_{8}{ }^{+}$ & $\mathrm{C}_{4} \mathrm{H}_{8}-$ Loss $^{*}$ & 10.69 & -8.61 \\
\hline 79 & $\mathrm{C}_{6} \mathrm{H}_{7}^{+}$ & ${ }^{-} \mathrm{C}_{4} \mathrm{H}_{9}-\operatorname{loss}$ & & \\
\hline 78 & $\mathrm{C}_{6} \mathrm{H}_{6}{ }^{++}$ & $\mathrm{C}_{4} \mathrm{H}_{10}$-loss & & \\
\hline
\end{tabular}

\subsection{Computational results}

The adamantane molecule has a highly symmetric structure, belonging to the $T_{\mathrm{d}}$ point group. Upon ionisation, the molecule is affected by Jahn-Teller distortion ${ }^{33,34}$ along the $C_{3}$ axis, leading to a structure of $C_{3 \mathrm{v}}$ symmetry (Fig. 1). The adiabatic ionisation energy is calculated to be $8.84 \mathrm{eV}$ (B3LYP/6-311++G(2d,p) including zero point energy corrections), in agreement with previous theoretical results, ${ }^{16}$ but slightly lower than the experimentally determined ionisation threshold (see Section 3.1).

Numerous pathways were investigated and only those relevant to the experimental findings, i.e., pathways with a rate limiting barrier that compares reasonably with the observed mechanism, are reported here. A summary of the adamantane cation PES containing only the rate limiting transition states leading to the observed carbon dissociation channels is presented in Fig. 5. The full PES landscape, the geometries of all transition and intermediate states and a detailed description of the different routes are available as ESI $\dagger$ to the interested reader. During the exploration of the PES, four intermediate states (int2, int8, int9 and int20) were found to be more stable than the adamantane cation by $0.07,0.07,0.49$ and $0.61 \mathrm{eV}$ respectively. Since their structures do not suggest high level of aromaticity, we performed calculations on the neutral counterparts of these intermediate states and found that they are all less stable than adamantane by $0.40,0.95,0.95$ and $0.88 \mathrm{eV}$, thus pointing to slightly lower ionisation energies.

Fragmentation channels involving carbon loss share the same initial two steps. First, the adamantane cation opens up to form int1. Next, a hydrogen atom shifts towards the $\mathrm{CH}_{2}$ dangling group (ts2), resulting in a boat-like $\mathrm{C}_{10} \mathrm{H}_{16}{ }^{+}$intermediate with a methyl group on top (int2) which is slightly more stable than the parent cation by $0.07 \mathrm{eV}$.

For the loss of a $\mathrm{C}_{3} \mathrm{H}_{6}$ unit, two energetically favourable pathways could be identified. Both paths involve a series of hydrogen shifts, eventually leading to formation of propene $\mathrm{C}_{3} \mathrm{H}_{6}$ and 3-methyl-1,4-cyclohexadiene cation $\left(\mathrm{C}_{7} \mathrm{H}_{10}{ }^{+}\right)(\mathrm{P} 1-1$ and P1-2) through transition states ts4 and ts7 (Fig. 5). The small energy difference between P1-1 and P1-2 is due to the slight change in orientation of $\mathrm{C}_{3} \mathrm{H}_{6}$ fragment with respect to $\mathrm{C}_{7} \mathrm{H}_{10}{ }^{+}$.

The $\mathrm{C}_{3} \mathrm{H}_{7}$-loss channel has a rate-limiting transition state ts5 at $10.02 \mathrm{eV}$ with respect to adamantane and the $\mathrm{C}_{3} \mathrm{H}_{7}$ fragment is lost as isopropyl radical (Fig. 5). A second path was found with a much higher $(10.90 \mathrm{eV})$ rate-limiting transition state (see Fig. S1, ESI $\dagger$ ). The former path likely dominates and it is the one represent in the overview figure (Fig. 5).

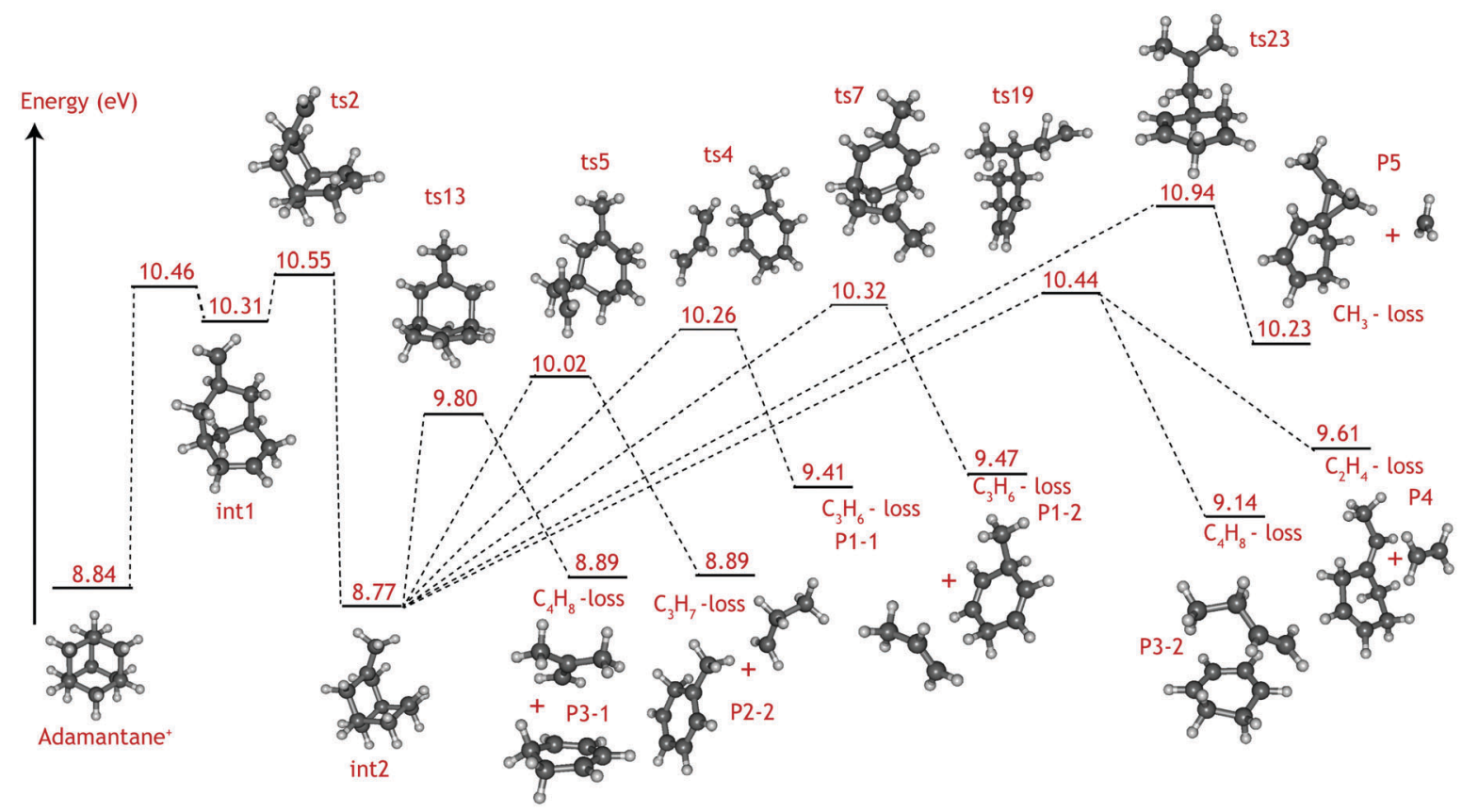

Fig. 5 Summary of the adamantane cation potential energy surface showing the rate limiting transition states leading to major dissociation channels. Energies are given in eV. The full PES, together with description of the steps and structures of transition and intermediate steps can be found in the ESI. $\dagger$ 
Three routes describing the $\mathrm{C}_{4} \mathrm{H}_{8}$-loss channel were found, all leading to the formation of 1,3-cyclohexadiene cation (Fig. S2 ESI $\dagger$ ); in two of these, the fragment is released as an isobutene unit (P3-1, Fig. 5) with rate-limiting transition states ts13 and ts23 at 9.80 and $10.62 \mathrm{eV}$ with respect to adamantane. The former route thus appears more favorable and has been included in Fig. 5. In the third route, the fragment is lost as 1-butene unit P3-2, with rate-limiting transition state ts19 at $10.44 \mathrm{eV}$. The energy difference between P3-1 and P3-2 is due to higher stability of the tertiary carbocation in isobutene.

As for the minor $\mathrm{CH}_{3}$ and $\mathrm{C}_{2} \mathrm{H}_{4}$ loss channels, rate limiting transition states at $10.94 \mathrm{eV}$ (ts23) and at $10.44 \mathrm{eV}$ (ts19) were found (Fig. 5).

Contrary to the carbon-loss channels, the loss of hydrogen proceeds via direct abstraction. Two non-equivalent $\mathrm{H}$ atoms, $\mathrm{H} 1$ and H2 (see Fig. 1), connected to tertiary and secondary $\mathrm{C}$ atoms, respectively, can be removed from adamantane ${ }^{+}$. The cleavage of $\mathrm{H} 1$ is calculated to require $1.58 \mathrm{eV}(10.42 \mathrm{eV}$ above adamantane), while $2.05 \mathrm{eV}$ is needed for removing $\mathrm{H} 2$ $(10.89 \mathrm{eV})$. These values are in good agreement with previous calculations. ${ }^{16,17}$ The removal of $\mathrm{H} 1$ is predicted to be an abstraction without reverse barrier.

\section{Statistical modelling}

The BD as well as the TOF distributions are modelled using the miniPEPICO program. ${ }^{23}$ This program uses the measured ionisation energy, transition state barriers as well as normal mode frequencies of the ground state of neutral adamantane, ground state ionic adamantane and rate limiting transition states as input for a RRKM model to simulate the experimental data. The program fits both the relative contributions of the different products (i.e. the breakdown diagram) and the peak shapes in the TPEPICO mass spectra (dissociation rates).

Computationally, the formation of 1-adamantyl is found to be lower in energy than the formation of 2-adamantyl. H-loss comprises an abstraction reaction without a reverse barrier and so no true transition state is located for this product. Hence, the transition state was estimated by optimizing the structure with a C-H distance of $4 \AA$ and was used for modelling the dissociative ionisation.

The $\mathrm{C}_{2} \mathrm{H}_{4}, \mathrm{C}_{3} \mathrm{H}_{x}$, and $\mathrm{C}_{4} \mathrm{H}_{y}$-loss channels were found to share a common rate limiting transition state ts2 (Fig. 5), which is associated with the creation of a boat-like $\mathrm{C}_{10} \mathrm{H}_{16}{ }^{+}$intermediate from the initial opening of the adamantane structure. Ts2 is located at $10.55 \mathrm{eV}$ with respect to the neutral adamantane. The $\mathrm{CH}_{3}$-loss channel is a minor, yet clearly observable product. Computationally it is not found to share the same rate limiting step as the other carbon loss pathways. Instead, its rate limiting transition state (ts23) is located at considerably higher energy $(10.94 \mathrm{eV})$ than those leading to the other observed products.

The fact that the loss of $\mathrm{C}_{3} \mathrm{H}_{7}, \mathrm{C}_{4} \mathrm{H}_{8}$ and $\mathrm{C}_{2} \mathrm{H}_{4}$ share the same rate limiting transition is implicitly taken into account in the RRKM model. The barriers for $\mathrm{CH}_{3}$ and $\mathrm{H}$-loss are used as fit parameters, while the common barrier is varied such that the error in the fit is minimised. The normal mode frequencies of the common transition state leading to $\mathrm{C}_{3} \mathrm{H}_{7}, \mathrm{C}_{4} \mathrm{H}_{8}$ and $\mathrm{C}_{2} \mathrm{H}_{4}$ are treated independently and are used as fit parameters that statistically determine the branching amongst these channels. In other words, in our model, the branching is determined at the initial transition state by the different phase space volumes belonging to the parallel dissociation pathways. In reality, it is likely that a sizeable fraction of the trajectories explores different dissociation pathways before product formation, giving rise to at least a partial equilibrium past the rate determining transition state. However, our model reproduces the experimental branching ratios quite well, meaning that our assumption is at least phenomenologically correct.

The results of the model fit are plotted as solid lines on top of the experimental points in Fig. 3 and 4. The model considers only the formation of the main product peaks and their ${ }^{13} \mathrm{C}$ isotopes. A reasonable agreement is found between the TOF and BD model and the experimental data. The resulting $0 \mathrm{~K}$ appearance energies and entropies of activations are summarised in Table 1.

\section{Discussion}

The VUV dissociative ionisation of adamantane is studied using a combination of experiments and quantum chemical computations. It is found that adamantane cations dissociate via a number of parallel channels, resulting in cations of $\mathrm{C}_{10} \mathrm{H}_{15}{ }^{+}, \mathrm{C}_{9} \mathrm{H}_{13}{ }^{+}, \mathrm{C}_{8} \mathrm{H}_{12}{ }^{+}, \mathrm{C}_{7} \mathrm{H}_{m}{ }^{+}(m=9,10,11)$ and $\mathrm{C}_{6} \mathrm{H}_{n}{ }^{+}$ $(n=6,7,8,9)$ composition. The $\mathrm{CH}_{3}$ and $\mathrm{C}_{2} \mathrm{H}_{4}$-loss channels comprise the weakest and the formation of $\mathrm{C}_{7} \mathrm{H}_{m}{ }^{+}\left(\mathrm{C}_{3} \mathrm{H}_{x}\right.$-loss $)$ comprises the strongest channel.

DFT computations confirm the experimental suggestion that $\mathrm{C}_{2} \mathrm{H}_{4}, \mathrm{C}_{3} \mathrm{H}_{x}$ and $\mathrm{C}_{4} \mathrm{H}_{y}$-loss channels share the same rate limiting transition state (ts2), which is characterised as $\mathrm{H}$-migration to create a methyl group, following by an opening of the adamantane cage and is located at $10.55 \mathrm{eV}(1.71 \mathrm{eV})$ with respect to adamantane (cation). The formed intermediate (int2) subsequently isomerises via submerged barriers to the detected products.

The lowest submerged barrier is ts 13 at $9.80 \mathrm{eV}$ and leads to isobutene $+1,3$-cyclohexadiene ${ }^{+}$. The second lowest pathway has a submerged barrier at $10.02 \mathrm{eV}$ (ts5) and results in $\mathrm{C}_{3} \mathrm{H}_{7}$-loss. The computed barrier heights appear to be at odds with the experimental work, which indicates that the $\mathrm{C}_{3} \mathrm{H}_{7}$ loss is the most abundant product channel. However, the finding could point to ts13 being a tighter transition state than ts5, causing the rate of the $\mathrm{C}_{3} \mathrm{H}_{7}$-loss channel to be significantly faster. Based on DFT harmonic frequencies, the calculated entropies of activation at $1000 \mathrm{~K}\left(\Delta S^{\ddagger}\right)$ for reactions int2 $\rightarrow$ ts5 and int $2 \rightarrow$ ts13 are $\Delta S^{\ddagger}=45.97 \mathrm{~J}(\mathrm{~mol} \mathrm{~K})^{-1}$ and $8.55 \mathrm{~J}(\mathrm{~mol} \mathrm{~K})^{-1}$, respectively, confirming the tighter nature of ts13. The RRKMderived activation entropies for the overall dissociation reaction agree with this picture, i.e. the transition state leading to $\mathrm{C}_{3} \mathrm{H}_{7}-$ loss is looser and the rate constant is a steeper rising function of energy above the threshold (see Table 1). 
Methyl and ethyl loss also proceed by opening the adamantane ring structure (ts2 at $10.55 \mathrm{eV}$ ). The rate limiting submerged barrier yielding $\mathrm{C}_{2} \mathrm{H}_{4}$ is located at $10.44 \mathrm{eV}$, which is significantly higher than those leading to the loss of $\mathrm{C}_{3} \mathrm{H}_{7}$ or $\mathrm{C}_{4} \mathrm{H}_{8}$. Looking at Fig. S2 in the $\mathrm{ESI}^{\dagger}$ it is seen that, according to theory, the $\mathrm{C}_{2} \mathrm{H}_{4}$-loss channel (ts22) competes with a low-barrier isomerisation channel (ts20). The entropies of activation, calculated from the theoretical DFT frequencies, of the two reactions $(-48.80$ and $-28.87 \mathrm{~J}(\mathrm{~mol} \mathrm{~K})^{-1}$, respectively), combined with the energy barriers ( 0.63 and $0.04 \mathrm{eV}$, respectively) suggest that isomerisation is the dominant reaction and only a small amount of int19 will lead to $\mathrm{C}_{2} \mathrm{H}_{4}$-loss. This finding is in excellent agreement with the smaller branching ratio to the $\mathrm{C}_{2} \mathrm{H}_{4}$-loss observed experimentally.

Demethylation is limited by a larger barrier (ts23) located at $10.94 \mathrm{eV}$. The relative abundance of the methyl and ethyl loss (Fig. 3) can be explained by the difference in entropy of activation derived from the RRKM modelling for the two reactions; ts23 is a looser transition state than ts19 with $\Delta S^{\ddagger}=11.25 \mathrm{~J}(\mathrm{~mol} \mathrm{~K})^{-1}$ compared to $\Delta S^{\ddagger}=-22.14 \mathrm{~J}(\mathrm{~mol} \mathrm{~K})^{-1}$, causing it to compete favorably.

The loss of an $\mathrm{H}$-atom is found, in agreement with prior studies, to yield 1-adamantyl via a reaction without a reverse barrier at an energy of $1.58 \mathrm{eV}$ with respect to the adamantane cation.

\section{Astrophysical implications}

Diamondoids are elusive components of the interstellar dust family; while abundant in presolar grains, only a handful of sources, mostly young stars with dusty disks, have robust detections of nanodiamonds spectral features, whose strength span 1-2 order of magnitudes. ${ }^{40}$ In these sources, diamondoids emission is present very close to the central star, where the radiation field is stronger; on the other side emission from 2-D hydrocarbons like Polycyclic Aromatic Hydrocarbons (PAHs) are more intense and widespread in the disk, even at larger distances. ${ }^{11,20}$ So far, the reason for this paucity has been identified in the very special physical conditions leading to the in situ formation of diamondoids. ${ }^{20}$ In these astrophysical environments, PAHs and diamondoids are subject to radiation fields that may ionise and/or photodissociate them.

Our findings show that adamantane dissociates mostly through the loss of $\mathrm{H}$ and $\mathrm{C}_{x} \mathrm{H}_{y}$ units, yielding small hydrocarbons. The dissociation channels can be compared to those of PAHs, often detected in conjunction with diamondoids in young stars, but much more ubiquitous in the ISM. The binding energy for carbon-loss channel in adamantane cation is found to be $1.85 \mathrm{eV}$ while for a PAH molecule with the same number of atoms, pyrene $\mathrm{C}_{16} \mathrm{H}_{10}{ }^{+}$, this number is $6.00 \mathrm{eV}{ }^{41}$ For the $\mathrm{H}$-loss channel the experimental binding energies are $1.66 \mathrm{eV}$ and $4.6 \mathrm{eV}^{42}$ for adamantane and pyrene, respectively. Thus adamantane appears to be less photostable than pyrene, as can be expected for a saturated hydrocarbon when compared to an aromatic one. Naturally the efficiency of dissociation will depend on the strength of the radiation source, e.g. the central star, and on the photo-absorption cross-section of the diamondoids. We speculate that the lower photostability together with the proposed very specific physical conditions needed for diamondoid formation may help explaining the very few detections of this material in astrophysical environments. The result of this work can be used as a key ingredient in photochemical modelling of nanodiamonds in typical sources like HD 97048 to test this hypothesis. Some questions still remain: do larger nanodiamonds share the same fragmentation behaviour with comparable binding energies? If so, what size of diamondoids would then be stable enough to survive close to the central radiation source? This clearly calls for further experimental studies on larger systems of astrophysical interest Finally, the effect of diamondoid dissociation may be evident in astronomical spectra; it is expected that upon dissociation, the strength of the typical $\mathrm{C}-\mathrm{H}$ stretch bands of nanodiamonds at 3.43 and $3.53 \mu \mathrm{m}$ will decrease, possibly favouring the appearance of aromatic $\mathrm{C}-\mathrm{H}$ stretches at $3.30 \mu \mathrm{m}$ as seen in observations. ${ }^{20}$

\section{Conflicts of interest}

There are no conflicts to declare.

\section{Acknowledgements}

AC and JB acknowledge the Netherlands Organisation for Scientific Research (Nederlandse Organisatie voor Wetenschappelijk Onderzoek, NWO) for VENI grants (grant number 639.041.543 and 722.013.014, respectively). AB and $\mathrm{PH}$ acknowledge funding by the Swiss Federal Office of Energy (BFE Contract Number SI/501269-01). Studies of interstellar molecules at the Leiden Observatory are supported through an advanced European Research Council grant 246976 and a Spinoza award. Calculations were carried out on the Dutch national e-infrastructure (Cartesius) with the support of SURF Cooperative, under project NWO EW project SH-362-15.

\section{References}

1 H. Schwertfeger, A. Fokin and P. Schreiner, Angew. Chem., 2008, 47, 1022-1036.

2 W. A. Clay, J. E. P. Dahl, R. M. K. Carlson, N. A. Melosh and Z. X. Shen, Rep. Prog. Phys., 2015, 78, 21.

3 J. Reiser, E. McGregor, J. Jones, R. Enick and G. Holder, Fluid Phase Equilib., 1996, 117, 160-167.

4 J. E. Dahl, S. G. Liu and R. M. K. Carlson, Science, 2003, 299, 96-99.

5 R. S. Lewis, T. Ming, J. F. Wacker, E. Anders and E. Steel, Nature, 1987, 326, 160-162.

6 E. Anders and E. Zinner, Meteoritics, 1993, 28, 490-514.

7 A. P. Jones, L. B. d'Hendecourt, S.-Y. Sheu, H.-C. Chang, C.-L. Cheng and H. G. M. Hill, Astron. Astrophys., 2004, 416, 235-241.

8 W. C. Saslaw and J. E. Gaustad, Nature, 1969, 221, 160-162. 9 T. Henning and F. Salama, Science, 1998, 282, 2204. 
10 O. Guillois, G. Ledoux and C. Reynaud, Astrophys. J., 1999, 521, L133-L36.

11 C. Van Kerckhoven, A. G. G. M. Tielens and C. Waelkens, Astron. Astrophys., 2002, 384, 568-584.

12 O. Pirali, M. Vervloet, J. E. Dahl, R. M. K. Carlson, A. G. G. M. Tielens and J. Oomens, Astrophys. J., 2007, 661, 919-925.

13 C. J. Bauschlicher, Y. Liu, A. Ricca, A. L. Mattioda and L. J. Allamandola, Astrophys. J., 2007, 671, 458.

14 J. Oomens, N. Polfer, O. Pirali, Y. Ueno, R. Maboudian, P. W. May, J. Filik, J. E. Dahl, S. Liu and R. M. Carlson, J. Mol. Spectrosc., 2006, 238, 158-167.

15 K. Lenzke, L. Landt, M. Hoener, H. Thomas, J. E. Dahl, S. G. Liu, R. M. K. Carlson, T. Moller and C. Bostedt, J. Chem. Phys., 2007, 127, 084320.

16 M. Steglich, F. Huisken, J. E. Dahl, R. M. K. Carlson and T. Henning, Astrophys. J., 2011, 729, 91.

17 N. Polfer, B. G. Sartakov and J. Oomens, Chem. Phys. Lett., 2004, 400, 201-205.

18 A. J. Lu, B. C. Pan and J. G. Han, Phys. Rev. B: Condens. Matter Mater. Phys., 2005, 72, 9.

19 R. J. Waltman and A. C. Ling, Can. J. Chem., 1980, 58, 2189-2195.

20 M. Goto, T. Henning, A. Kouchi, H. Takami, Y. Hayano, T. Usuda, N. Takato, H. Terada, S. Oya, C. Jäger and A. C. Andersen, Astrophys. J., 2009, 693, 610-616.

21 M. Johnson, A. Bodi, L. Schulz and T. Gerber, Nucl. Instrum. Methods Phys. Res., 2009, 610, 597-603.

22 A. Bodi, M. Johnson, T. Gerber, Z. Gengeliczki, B. Sztaray and T. Baer, Rev. Sci. Instrum., 2009, 80, 034101.

23 B. Sztaray, A. Bodi and T. Baer, J. Mass Spectrom., 2010, 45, 1233-1245.

24 B. Sztaray and T. Baer, Rev. Sci. Instrum., 2003, 74, 3763-3768.

25 M. J. Frisch, G. W. Trucks, H. B. Schlegel, G. E. Scuseria, M. A. Robb, J. R. Cheeseman, G. Scalmani, V. Barone, B. Mennucci, G. A. Petersson, H. Nakatsuji, M. Caricato, X. Li, H. P. Hratchian, A. F. Izmaylov, J. Bloino, G. Zheng, J. L. Sonnenberg, M. Hada, M. Ehara, K. Toyota, R. Fukuda, J. Hasegawa, M. Ishida, T. Nakajima, Y. Honda, O. Kitao, H. Nakai, T. Vreven, J. A. Montgomery, Jr., J. E. Peralta, F. Ogliaro, M. Bearpark, J. J. Heyd, E. Brothers, K. N. Kudin, V. N. Staroverov, R. Kobayashi, J. Normand, K. Raghavachari, A. Rendell, J. C. Burant, S. S. Iyengar, J. Tomasi, M. Cossi, N. Rega, J. M. Millam, M. Klene,
J. E. Knox, J. B. Cross, V. Bakken, C. Adamo, J. Jaramillo, R. Gomperts, R. E. Stratmann, O. Yazyev, A. J. Austin, R. Cammi, C. Pomelli, J. W. Ochterski, R. L. Martin, K. Morokuma, V. G. Zakrzewski, G. A. Voth, P. Salvador, J. J. Dannenberg, S. Dapprich, A. D. Daniels, O. Farkas, J. B. Foresman, J. V. Ortiz, J. Cioslowski and D. J. Fox, Gaussian 09, Revision D.01, Gaussian Inc., Wallingford CT, 2009.

26 C. Peng and H. Bernhard Schlegel, Isr. J. Chem., 1993, 33, 449-454.

27 C. Peng, P. Y. Ayala, H. B. Schlegel and M. J. Frisch, J. Comput. Chem., 1996, 17, 49-56.

28 A. Allouche, J. Comput. Chem., 2011, 32, 174-182.

29 A. Mozhayskiy and A. I. Krylov, ezSpectrum, 2009, Version 3.0, http://iopenshell.usc.edu/downloads.

30 T. Baer and W. Hase, Unimolecular Reaction Dynamics: Theory and Experiments: Theory and Experiments, Oxford University Press, USA, 1996.

31 NIST, NIST Chemistry WebBook, National Institute of Standards and Technology, Gaithersburg MD, 20899, 2009, NIST Standard Reference Database Number 69.

32 W. Schmidt, Tetrahedron, 1973, 29, 2129-2213.

33 A. Patzer, M. Schütz, T. Möller and O. Dopfer, Angew. Chem., 2012, 51, 4925-4929.

34 M. E. Crestoni and S. Fornarini, Angew. Chem., 2012, 51, 7373-7375.

35 S. D. Worley, G. D. Mateescu, C. W. McFarland, R. C. Fort and C. F. Sheley, J. Am. Chem. Soc., 1973, 95, 7580-7586.

36 J. Bouwman, B. Sztaray, J. Oomens, P. Hemberger and A. Bodi, J. Phys. Chem. A, 2015, 119, 1127-1136.

37 B. West, C. Joblin, V. Blanchet, A. Bodi, B. Sztaray and P. M. Mayer, J. Phys. Chem. A, 2012, 116, 10999-11007.

38 P. Hemberger, A. Bodi, T. Gerber, M. Wuertemberger and U. Radius, Chem. - Eur. J., 2013, 19, 7090-7099.

39 P. Hemberger, A. Bodi, J. H. J. Berthel and U. Radius, Chem. - Eur. J., 2015, 21, 1434-1438.

40 B. Acke and M. E. van den Ancker, Astron. Astrophys., 2006, 457, 171-181.

41 B. West, F. Useli-Bacchitta, H. Sabbah, V. Blanchet, A. Bodi, P. M. Mayer and C. Joblin, J. Phys. Chem. A, 2014, 118, 7824-7831.

42 Y. Ling, Y. Gotkis and C. Lifshitz, Eur. J. Mass Spectrom., 1995, 1, 41-49. 